\section{Visual attention to facial features following emotion remediation in schizophrenia}

\section{P Marsh', T RusselI', M Green ${ }^{2,3}$, M Coltheart ${ }^{1}$}

${ }^{1}$ Macquarie Centre for Cognitive Science, Macquarie University; ${ }^{2}$ School of Psychiatry, University of New South Wales; and ${ }^{3}$ The Black Dog Institute, Sydney, Australia

Background: Considerable evidence attests to impaired facial emotion perception in schizophrenia, alongside abnormalities of attention evident in restricted scanning and avoidance of facial features (eyes, nose and mouth). Recent studies have successfully improved social cognition in schizophrenia with the use of targeted remediation techniques. In this study, we used a computer-based microexpression training tool (METT; Ekman, 2003) with concurrent assessment of eye movements to investigate changes in visual attention to faces following emotion remediation in schizophrenia.

Methods: Twenty-six participants with schizophrenia were exposed to active training using the METT, while 14 were assigned to a repeated-exposure group. Training comprised video clips with verbal commentary detailing the difference between frequently confused emotions and directing attention to relevant facial features, and practice with feedback regarding accuracy. The repeated-exposure group viewed the video clips but were not exposed to the verbal commentary or feedback during practice. Affect recognition and concurrent eye movement recordings were collected preand posttraining in both groups to test the effects of training.

Results: Active remediation training improved emotion recognition accuracy, while repeated exposure to visual stimuli did not. Participants who received METT training showed a general increase in attention to feature areas of faces posttraining. For specific facial expressions, attention to feature areas increased or decreased according to the instructions given during remediation.

Conclusions: Training with the METT improves emotion-processing skills in schizophrenia, while repeated exposure does not. Visual attention to facial features that are important for distinguishing between facial expressions improves with METT remediation.

\section{ADHD and first-episode schizophrenia show distinct scanpaths to emotional faces}

\section{PJ Marsh', AW Harris ${ }^{2,3}$, S Clarke ${ }^{3}$, M Kohn $^{4}$, I Lazzaro², K Brown ${ }^{2.5}$, M Charles ${ }^{6}$, C Latimer ${ }^{6}$, LM Williams²}

${ }^{1}$ Macquarie Centre for Cognitive Science, Macquarie University; ${ }^{2}$ The Brain Dynamics Centre; ${ }^{3}$ Westmead Hospital; "Westmead Children's Hospital;

${ }^{5} J o i n t$ Centres of Mental Health Excellence, Westmead Hospital; and

${ }^{6}$ School of Psychology, University of Sydney, Sydney, Australia

Background: Attention deficit hyperactivity disorder (ADHD) and early-onset schizophrenia are both marked by significant deficits in social and emotional functioning. In this study, we tracked visual scanpaths while participants passively viewed emotional faces to examine the processing of facial emotions in patients with ADHD and first-episode schizophrenia (FES).

Methods: A corneal reflection technique was used to record eye fixations and saccades in 42 ADHD, 20 FES and 37 matched healthy control participants, between 12 and 18 years of age. Visual scanpaths were recorded while participants viewed happy and sad facial expressions, as well as neutral and nonface control stimuli.

Results: FES participants, compared with both ADHD and control participants, were distinguished by 'restricted' scanpaths (eg reduced duration and number of fixations and reduced dispersal of fixations), as well as reduced attention to the salient features of positive and negative facial expressions. Conversely, ADHD participants exhibited more dispersed fixations compared with controls, although differences were only significant for the neutral face.

Conclusions: Our results suggest that FES and ADHD may be distinguished by at least partially separable deficits in facial emotion processing. While the abnormal scanpaths of FES may reflect an inability to form an integrated gestalt of salient facial features, ADHD might, to some extent, be characterized by an inability to disengage from nonfeature (task irrelevant) facial areas. 